\section{SAMHDI prive le VIH des nucléotides essentiels à la synthèse d'ADN viral}

Hichem Lahouassa, Loïc Dragin, Catherine Transy, Florence Margottin-Goguet
Inserm U1016, Institut Cochin, 22, rue Méchain, 75014 Paris, France. florence.margottin-goguet@inserm.fr

le taux de nucléotides reste limitant pour l'infection des cellules par ce virus. Ainsi, Vpx d'un côté et les dN de l'autre, augmentent l'infection par le VIH-l.

L'ensemble de ces observations nous a conduits à explorer l'hypothèse selon laquelle Vpx pourrait augmenter la concentration de dNTP intracellulaires. Dans un premier temps, nous avons donc cherché à savoir si l'addition de $\mathrm{dN}$ au milieu de culture de macrophages cultivés in vitro permettrait de restaurer l'infection de ces cellules par le SIV (un virus proche du VIH2) dépourvu du gène codant pour Vpx. En d'autres termes : les dN permettent-ils à un virus SIV de s'affranchir de Vpx? La réponse est oui, mais partiellement. Ainsi, pour la première fois, des conditions métaboliques particulières permettaient à un virus SIV d'infecter des macrophages en l'absence de Vpx. Par la suite, une quantification directe de la concentration des dNTP intracellulaires a permis de montrer que Vpx augmentait significativement le taux des quatre dNTP (dATP, dCTP, dGTP et dTP) dans les macrophages.

La protéine SAMHDI est une dNTPase dépendante du dGTP

En 2011, la protéine SAMHDI (impliquée dans le syndrome d'Aicardi-Goutières ${ }^{1}[7]$ ) pas leur ADN nucléaire, contrairement aux lymphocytes T qui prolifèrent [6]. Les VIH se sont d'ailleurs adaptés à ces conditions de faible concentration en nucléotides en développant des RT de très forte affinité vis-à-vis des dNTP [6]. II n'en reste pas moins que l'addition de désoxynucléosides $(\mathrm{dN})$, convertis en dNTP dans la cellule, permet d'augmenter fortement l'infection des macrophages par le $\mathrm{VIH}-1$, indiquant que

\footnotetext{
Le syndrome d'Aicardi-Goutières (AGS) est une encéphalopathie subaiguë à transmission mendélienne, associant classiquement des calcifications des noyaux gris centraux, une leucodystrophie et une lymphocytose du liquide céphalo-rachidien. Un peu plus de 120 cas ont été publiés dans la littérature. Les symptômes débutent [...] dans les premiers jours ou mois de vie, sous forme d'une encéphalopathie subaiguë sévère associée à une épilepsie (53\%), des engelures des extrémités ( $43 \%$ ) et des épisodes fébriles aseptiques (40\%). Les symptômes évoluent sur quelques mois (apparition d'une microcéphalie et d'un syndrome pyramidal) avant de se stabiliser. (source Orphanet).
}

Le taux cellulaire de dNTP : un élément clé pour l'infection du VIH

Le travail a commencé avec l'étude de la protéine auxiliaire Vpx. Alors que les VIH-1 et VIH-2, comme tous les rétrovirus, partagent l'expression de protéines structurales et catalytiques codées par les gènes gag, pol et env, ils diffèrent par leur équipement en protéines auxiliaires. Ainsi, 


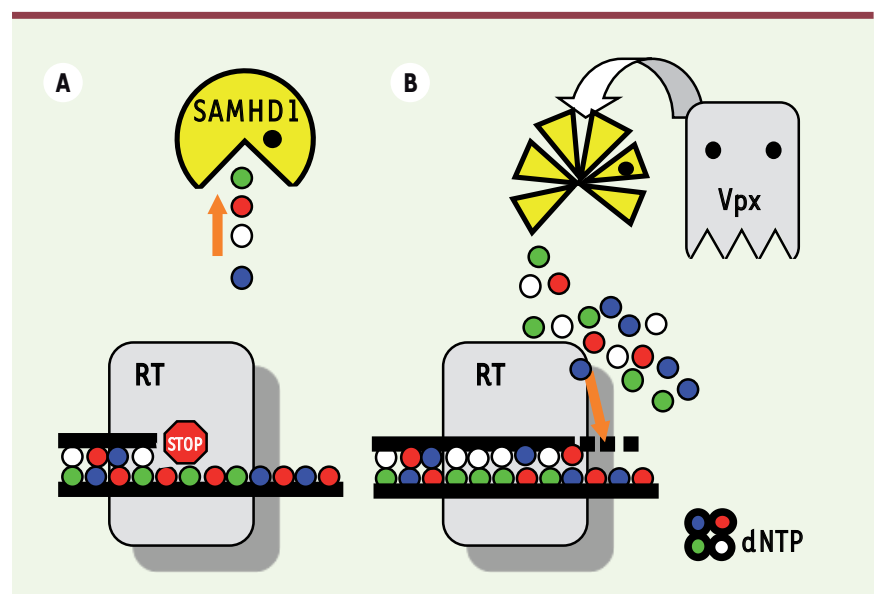

Figure 1. Rôle du facteur de restriction SAMHDI et de la protéine virale Vpx dans la régulation de la concentration cellulaire de ANTP. A. Dans les cellules monocytaires, les dNTP sont maintenus à une concentration très faible, du fait de leur dégradation par la protéine SAMHDI. En absence de substrat (dNTP), la transcriptase inverse (RT) du virus ne peut synthétiser l'ADN viral de façon optimale; la réplication virale est bloquée à une étape précoce. Les VIH-1 et VIH-2/SIVsm sont tous les deux sensibles à l'effet inhibiteur de SAMHDI. B. Le VIH-2 et le virus simien apparenté (SIVsm) ont développé une arme contre SAMHDI : la protéine virale Vpx. En dégradant SAMHDI, Vpx augmente le taux intracellulaire de dNTP, ce qui permet une synthèse efficace de l'ADN viral et l'infection des cellules. était identifiée, non seulement comme une protéine limitant l'infection par les VIH-l et VIH-2/SIVsm, mais aussi comme une cible cellulaire dégradée en présence de Vpx (un mécanisme viral permettant de contrecarrer l'activité de ce facteur de restriction) [1, 2]. Cette découverte était-elle compatible avec un rôle direct de Vpx sur le taux de dNTP intracellulaire? Deux caractéristiques de SAMHDI ont alors revêtu une importance particulière à nos yeux : son domaine HD, un domaine associé au métabolisme des acides nucléiques, et son homologie de séquence avec des enzymes permettant l'hydrolyse des dNTP (ou dNTPases) chez les bactéries. Nous nous sommes donc demandé si SAMHDl était une enzyme de type dNTPase, auquel cas sa dégradation en présence de Vpx pouvait conduire à une augmentation du taux de dNTP intracellulaire. Pour tester cette hypothèse, nous avons, d'une part, supprimé son expression dans une lignée monocytaire (cellules THP-1 [human acute monocytic leukemia cell line]) puis quantifié les dNTP et, d'autre part, exprimé la protéine dans des cellules humaines qui ne l'expriment pas de façon naturelle (cellules U937), puis quantifié les dNTP. Nous avons observé que l'absence de SAMHDI mimait l'effet de Vpx, c'est-à-dire entraînait une augmentation du taux de dNTP, tandis que sa surexpression diminuait de façon drastique le taux de désoxynucléotides, et ce seulement si le domaine HD était intact. Par ailleurs la protéine SAMHDI, produite chez la bactérie et purifiée, est capable d'hydrolyser les dNTP in vitro, plus précisément le dGTP, mais aussi les trois autres nucléotides en présence de dGTP. SAMHDI est donc une dNTPase dépendante du dGTP $[3,8,9]$.

\section{Conclusions et perspectives}

SAMHDl est un régulateur clé du métabolisme des désoxynucléotides, qui limite l'infection par les VIH en maintenant un taux de dNTP dans les macrophages trop faible pour permettre un fonctionnement optimal de la transcriptase inverse (Figure IA). Le VIH-2 (ou le SIVsm) a développé une arme pour se défendre de l'activité de SAMHDl, la protéine Vpx. Cette dernière induit la dégradation de SAMHDl et ainsi augmente le taux intracellulaire de dNTP (Figure 1B). Le VIH-1, quant à lui, est sensible à SAMHDl mais échappe partiellement à son action, bien qu'il ne code pas pour Vpx.

La déplétion en désoxynucléotides apparaît donc comme une stratégie de défense contre les VIH, particulièrement « intelligente » dans les cellules quiescentes qui n'ont pas besoin d'un contenu élevé en dNTP. La modulation du taux de désoxynucléotides intracellulaires est certainement un axe de recherche à prendre en considération dans le cadre de l'élaboration de nouvelles stratégies contre les $\mathrm{VIH}$, mais peut-être aussi contre d'autres pathogènes. En effet, le mécanisme de déplétion en désoxynucléotides pourrait être utilisé par la cellule pour résister à l'infection contre différents pathogènes dont la survie dépend d'une synthèse d'ADN dans des cellules quiescentes. $\diamond$
SAMHD1 deprives HIV of nucleotides, essential for viral DNA synthesis

\section{LIENS D'INTÉRÊT}

Les auteurs déclarent n'avoir aucun lien d'intérêt concernant les données publiées dans cet article.

\section{RÉFÉRENCES}

1. Hrecka K, Hao C, Gierszewska M, et al. Vpx relieves inhibition of HIV-1 infection of macrophages mediated by the SAMHDl protein. Nature $2011 ; 474: 658-61$.

2. Laguette $N$, Sobhian $B$, Casartelli $N$, et al. SAMHD1 is the dendritic- and myeloid-cell-specific HIV-1 restriction factor counteracted by Vpx. Nature 2011 ; 474 : 654-7.

3. Lahouassa H, Daddacha W, Hofmann H, et al. SAMHDI restricts the replication of human immunodeficiency virus type 1 by depleting the intracellular pool of deoxynucleoside triphosphates. Nat Immunol 2012 ; 13 : 223-8.

4. Ayinde D, Maudet C, Transy C, Margottin-Goguet F. Limelight on two HIV/SIV accessory proteins in macrophage infection : is Vpx overshadowing Vpr? Retrovirology $2010 ; 7: 35$.

5. Goujon C, Riviere L, Jarrosson-Wuilleme L, et al. SIVSM/ HIV-2 Vpx proteins promote retroviral escape from a proteasome-dependent restriction pathway present in human dendritic cells. Retrovirology $2007 ; 4: 2$.

6. Diamond TL, Roshal M, Jamburuthugoda VK, et al. Macrophage tropism of HIV-l depends on efficient cellular dNTP utilization by reverse transcriptase. J Biol Chem $2004 ; 279$ : 51545-53.

7. Rice GI, Bond J, Asipu A, et al. Mutations involved in Aicardi-Goutieres syndrome implicate SAMHDI as regulator of the innate immune response. Nat Genet $2009 ; 41: 829-32$.

8. Goldstone DC, Ennis-Adeniran V, Hedden JJ, et al. HIV-1 restriction factor SAMHDl is a deoxynucleoside triphosphate triphosphohydrolase. Nature $2011 ; 480$ : 379-82.

9. Powell RD, Holland PJ, Hollis T, Perrino FW. AicardiGoutieres syndrome gene and HIV-1 restriction factor SAMHD1 is a dGTP-regulated deoxynucleotide triphosphohydrolase. J Biol Chem 2011 ; 286 : 43596-600

10. Marquet R, Guerrero S, Bernacchi S. Un facteur de transcription se fait complice du VIH-1 pour détruire les défenses cellulaires. Med Sci (Paris) 2012 ; $28: 356-8$. 